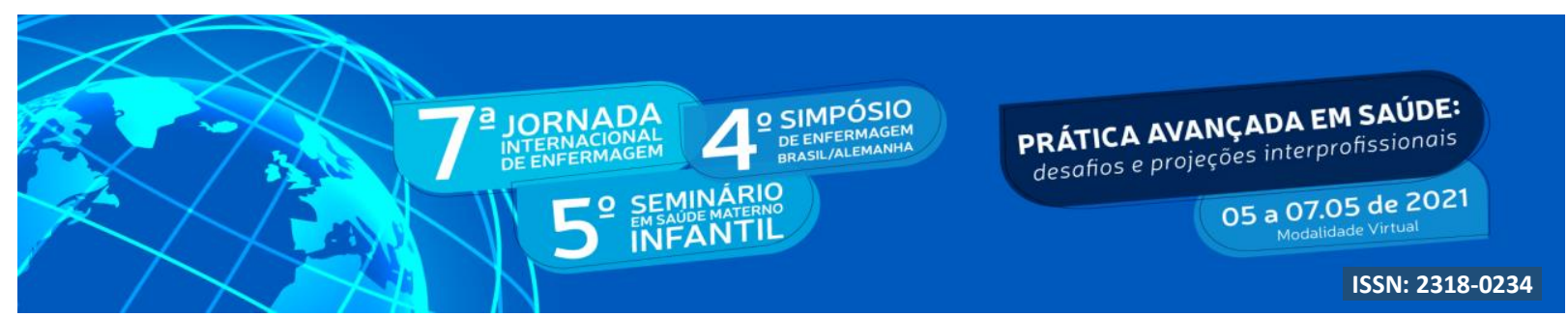

DOI: http://doi.org/10.48195/jie2021-007

\title{
INTERVENÇÃO DA ENFERMAGEM SOBRE A POLÍTICA NACIONAL DE DST/AIDS: RELATO DE EXPERIÊNCIA ${ }^{1}$
}

\section{Cláudia Ferrony Rivas²; Gabriella Dalla Corte Córdova ${ }^{3}$; Ravel Quevedo dos Santos ${ }^{4}$; Fabiana Porto da Silva ${ }^{5}$; Juliana Silveira Colomé ${ }^{6}$}

\begin{abstract}
RESUMO
Este estudo tem por objetivo relatar a experiência vivenciada por acadêmicos de Enfermagem da Universidade Franciscana-UFN a partir de uma entrevista e ação realizada sobre a "Política Nacional de DST/AIDS: Princípios e Diretrizes" no município de Santa Maria-RS. Trata-se de um relato de experiência de caráter descritivo integrando um dos processos de aprendizagem teórico-prática disponibilizada pela disciplina de Políticas Públicas em Saúde. No sentido de sistematizar o processo da entrevista e ação, os resultados serão descritos em três unidades temáticas, tais como: Detalhamento da coleta de dados; Detalhamento do processo de intervenção; e, Vivências significativas por parte dos autores do artigo. Conclui-se que nas realizações das atividades torna-se primordial a busca de métodos que incentivem e conscientizem a população em relação à prevenção das ISTs. Dessa forma, acreditamos que nossa intervenção possa contribuir positivamente gerando resultados gratificantes.
\end{abstract}

Palavras-chave: Enfermagem; Educação em saúde; Políticas Públicas.

\begin{abstract}
This study aims to report an experience lived by nursing students at the Franciscan University-UFN from an interview and action carried out on the "National Policy on STD / AIDS: Principles and Guidelines" in the city of Santa Maria-RS. This is a descriptive experience report integrating one of the theoretical-practical learning processes provided by the Public Health Policy discipline. In order to systematize the interview and action process, the results obtained in three thematic units, such as: Detailing of data collection; Details of the intervention process; and, Relevant experiences by the authors of the article. It is concluded that in the performance of activities, a search for methods that encourages and makes the population aware of the prevention of STIs becomes paramount. Thus, we believe that our intervention can contribute positively generating rewarding results.
\end{abstract}

Key Words: Nursing; Health Education; Public Policies.

\footnotetext{
${ }^{1}$ Trabalho da disciplina de Políticas Públicas em Saúde - Universidade Franciscana (UFN).

${ }^{2}$ Estudante do Curso de Enfermagem da Universidade Franciscana. claudiamfrivas@gmail.com

${ }^{3}$ Estudante do Curso de Enfermagem da Universidade Franciscana. gabrielladcc@ gmail.com

${ }^{4}$ Estudante do Curso de Enfermagem da Universidade Franciscana. raveldagalera@ gmail.com

${ }^{5}$ Docente do curso de Enfermagem da Universidade Franciscana (UFN). Responsável pela disciplina de Saúde Coletiva II. Orientadora do trabalho. fabianaporto54@gmail.com

${ }^{6}$ Docente do curso de Enfermagem da Universidade Franciscana (UFN). Responsável pela disciplina de Saúde

Coletiva II. Orientadora do trabalho. juliana@ufn.edu.br 1
} 


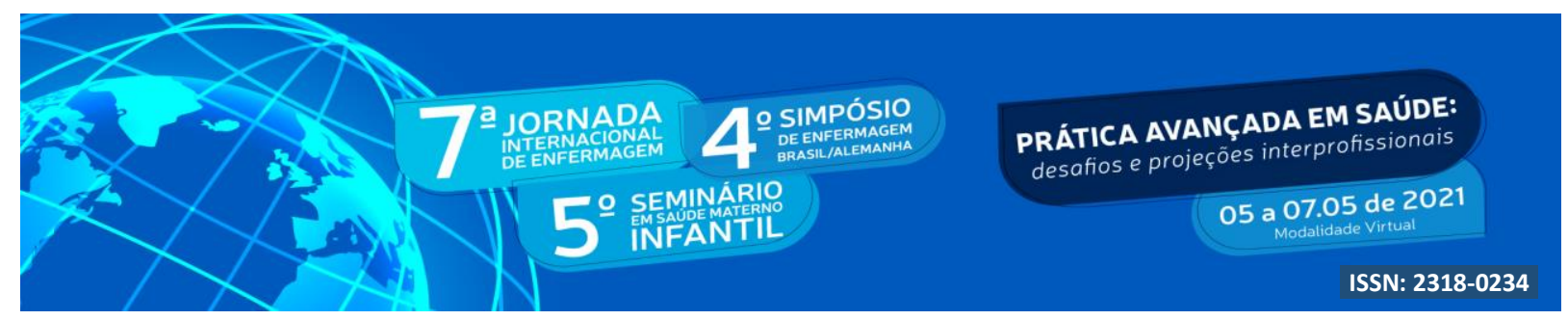

\section{INTRODUÇÃ̃O}

A "Política Nacional de DST/AIDS: Princípios e Diretrizes", criada em 1999, tem o objetivo de sistematizar a atuação para construir um instrumento que possibilite subsidiar as ações de saúde no âmbito da Secretaria de Políticas de Saúde do Ministério da Saúde. Dessa forma, o comprometimento de diferentes setores governamentais e da população torna-se fundamental para garantir o atendimento e os direitos dos usuários (BRASIL, 1999).

No ano de 2016 no Brasil, o Departamento de Vigilância, Prevenção e Controle das IST, do HIV/Aids e das Hepatites Virais, altera a nomenclatura de DTS (doença sexualmente transmissível) para IST (infecção sexualmente transmissível), tendo em vista que doença implica sinais e sintomas perceptíveis no organismo da pessoa, já as infecções podem ser assintomáticas e constatadas apenas através exames laboratoriais (BRASIL, 2016). Contudo, em relação a "Política Nacional de DST/AIDS: Princípios e Diretrizes" do ano de 1999 não houve nenhuma alteração/atualização da nomenclatura pelo Ministério da Saúde, diante disso ao abordar sobre esse documento será pelo título original.

No Brasil, as Infecções Sexualmente Transmissíveis (ISTs) com maior prevalência são a aids, sífilis, gonorreia, herpes genital e HPV. As formas de transmissão ocorrem, principalmente, por contato sexual, via sanguínea, transmissão vertical, parto e amamentação ocasionadas por 30 agentes etiológicos (FERREIRA et al., 2018). A população com maior vulnerabilidade em relação a essas infecções está na faixa etária dos 10 aos 24 anos, atingindo cerca de 54 milhões de pessoas (KRABBE et al., 2016).

As ISTs podem ser provocadas por vírus, fungos, protozoários e bactérias, se manifestando por meio de irritações, corrimentos, feridas nos órgãos genitais, dor no ato sexual, podendo causar complicações psicossociais e econômicas. Geralmente, o sexo feminino é mais suscetível às complicações. Além disso, o tratamento está relacionado ao acompanhamento multiprofissional, higiene pessoal e o uso de uma medicação correta (SANTOS; SILVA; FONTELES, 2017).

O Sistema Único de Saúde (SUS) prevê uma mudança no âmbito do cuidado priorizando uma visão holística do usuário. Contudo, ainda expressa no campo das ISTs um processo marcado pelo modelo biomédico onde as ações preventivas são limitadas e padronizadas culpabilizando o sujeito (ARAÚJO; SILVA; RODRIGUES, 2019). Nessa 


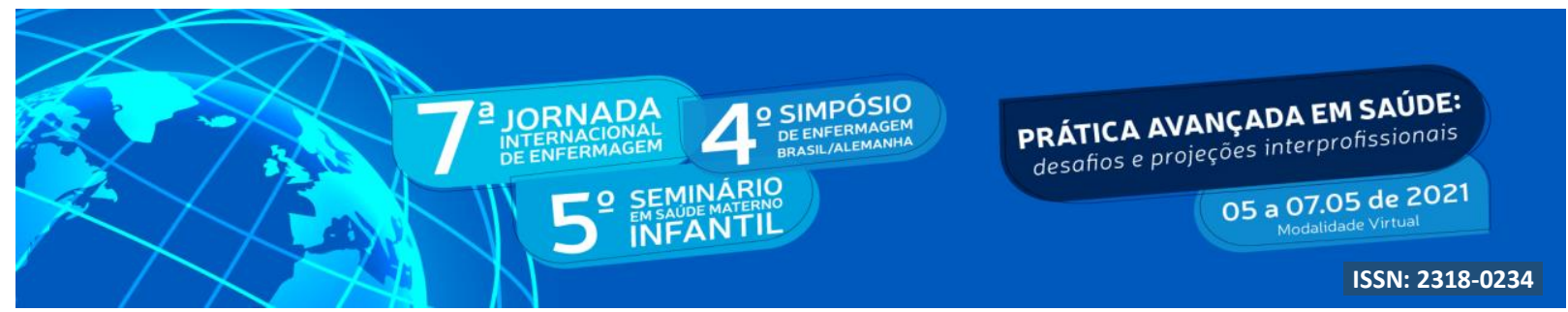

perspectiva, a enfermagem é protagonista de romper com modelo processo saúde/doença atentando às práticas de acolhimento, vínculo e humanização de acordo com as diretrizes e princípios do SUS.

De acordo com a Portaria $\mathrm{N}^{\circ} 1.625$ de 10 de julho de 2007, a consulta de enfermagem é uma das ações que auxiliam na promoção da saúde e prevenção de doenças com o propósito de realizar um plano de cuidados, obter diagnósticos e propagar informações de acordo com as necessidades de cada paciente. O compromisso do enfermeiro principalmente na equipe de Saúde da Família (ESF) é orientar, esclarecer dúvidas e prestar um cuidado mais seguro estimulando redução dos riscos e hábitos saudáveis (FERREIRA et al., 2018).

Com isso, manter uma comunicação adequada com a população em relação à sexualidade e as formas de prevenir as ISTs através do uso de preservativos e um acompanhamento anual com exames de rotina é imprescindível para evitar a contaminação e possíveis complicações (JESUS, 2018).

Diante do exposto, conhecer a "Política Nacional de DST/AIDS: Princípios e Diretrizes" compreendendo as atribuições, dificuldades e recursos possibilita uma melhor percepção das ações desenvolvidas no âmbito da saúde a fim de adquirir conhecimentos para gerar mudanças no modo de gerir e atuar.

\section{OBJETIVO}

Este estudo tem por objetivo relatar a experiência vivenciada por acadêmicos de Enfermagem da Universidade Franciscana-UFN a partir de uma entrevista e ação realizada sobre a "Política Nacional de DST/AIDS: Princípios e diretrizes" no município de Santa Maria-RS.

\section{METODOLOGIA}

Trata-se de um relato de experiência de caráter descritivo, a partir das vivências de acadêmicos de enfermagem do $4^{\circ}$ semestre da Universidade Franciscana (UFN) ocorrida no mês de junho de 2018, referente a uma entrevista e uma ação realizada pelos estudantes sobre a "Política Nacional de DST/AIDS: Princípios e diretrizes" no município de Santa Maria-RS. 


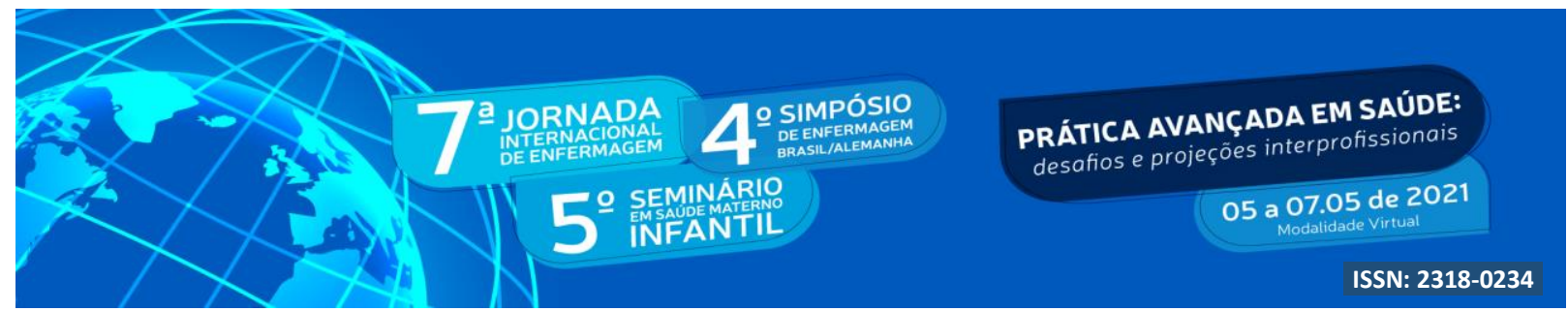

A experiência integrou um dos processos de aprendizagem teórico-prática disponibilizado pela disciplina de "Políticas Públicas em Saúde" com carga horária de 100 horas. Cujo objetivo geral da matéria é conhecer as políticas públicas em saúde vigentes no contexto brasileiro e suas implicações nas práticas assistenciais, na organização dos serviços e na gestão de programas e estratégias articuladas às necessidades individuais e coletivas de saúde da população.

Os relatos dos autores do artigo foram identificados como autor (A1), autor 2 (A2) e autor 3 (A3), para manter o anonimato dos mesmos. Salienta-se que o projeto não passou pelo comitê de ética, pelo fato de ser um relato de experiência elaborado com base nas reflexões e sentimentos vivenciados pelos acadêmicos ao estudar a temática e desenvolver a atividade com fins educativos e avaliativos para a disciplina do semestre.

Através da linha de conhecimento dos acadêmicos sobre o tema, foram formuladas questões abertas para a entrevista que ocorreu na Secretaria Municipal de saúde de Santa Maria-RS, em dia e horário previamente agendados, com a responsável pela gestão da política. Entre os questionamentos destacam-se perguntas sobre ações do setor, recursos, dificuldades encontradas e funcionamento que após foram transcritas para análise dos alunos.

Já a intervenção foi desenvolvida pelos estudantes a partir da percepção da necessidade de divulgação da prevenção das ISTs nos ambientes públicos da cidade como maneira de contribuir para a "Política Nacional de DST/AIDS: Princípios e Diretrizes" do município. Ocorreu nos banheiros públicos masculinos e femininos da Praça Saldanha Marinho, no Shopping Popular e em um bar que possui quartos privados no dia 13 de junho, vinculado à alusão ao dia dos namorados que ocorre no dia 12 de junho.

\section{RESULTADOS}

No sentido de sistematizar o processo da entrevista e ação, os resultados serão descritos em três unidades temáticas, tais como: Detalhamento da coleta de dados; Detalhamento do processo de intervenção; e, Vivências significativas por parte dos autores do artigo. 


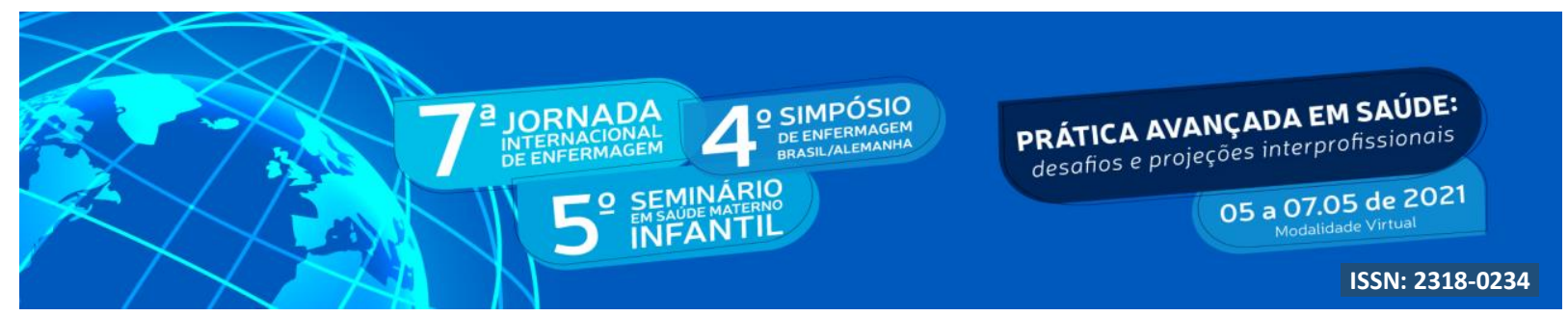

\subsection{Descrição da coleta de dados}

No primeiro dia da vivência, dia 27 de maio de 2019 foi realizada uma visita em dia e horário previamente agendados na Secretaria Municipal de Saúde de Santa Maria-RS, na qual foi observado o local de trabalho e dialogado com a responsável pela gestão da política e uma acadêmica de enfermagem que também estava presente. Sendo assim, foi realizado um questionário com oito perguntas relacionadas à importância, recursos, dificuldades, atendimentos, atividades realizadas, disponibilidade de medicações e em relação ao preconceito.

A responsável mencionou que ela começou a trabalhar com essa política em junho de 2018, com ações de levantamento de dados epidemiológicos priorizando alguns casos. Diante da incidência de Sífilis Congênita, criou o Comitê de Transmissão Vertical para investigar e notificar as ocorrências. Outra atividade feita é a linha de cuidados mediante ao HIV buscando descentralizar o atendimento que ainda é especializado. Relata a reestruturação e importância da Atenção Básica em fazer o acompanhamento e deixar casos mais graves aos serviços da Casa 13 de Maio (unidade de saúde foco no atendimento de ISTs).

Os projetos possuem recursos para os materiais de leitura, folders e preservativos. Afirma que muitas vezes faltam o repasse de materiais pelo Ministério da Saúde e o município tem que repor. Dificuldades na implementação ainda são uma preocupação, visto que muitos profissionais não desejam maior envolvimento em ações que estão fora das rotinas de seus trabalhos. Embora exista esse obstáculo, houve o crescimento da realização dos testes rápidos realizados nas Unidades de Saúde beirando cerca de 800 por mês.

Com a falta de criação de vínculo em diversas Unidades Básicas de Saúde (UBS), os usuários procuram ir a Casa 13 de Maio pelo fato de se sentirem acolhidos pela equipe multiprofissional composta por um psicólogo, assistente social, enfermeira que coordena o serviço, três médicos infectologistas, nutricionista com um turno na semana, dermatologista, ginecologista e clínica geral. A procura do sistema ocorre principalmente por jovens universitários. O atendimento ocorre de segunda a sexta-feira por livre demanda.

Foi abordado que a Profilaxia Pós-Exposição de Risco (PEP) é realizada nas Unidades de Pronto atendimento após acidente de trabalho, relação sexual desprotegida ou rompimento, 


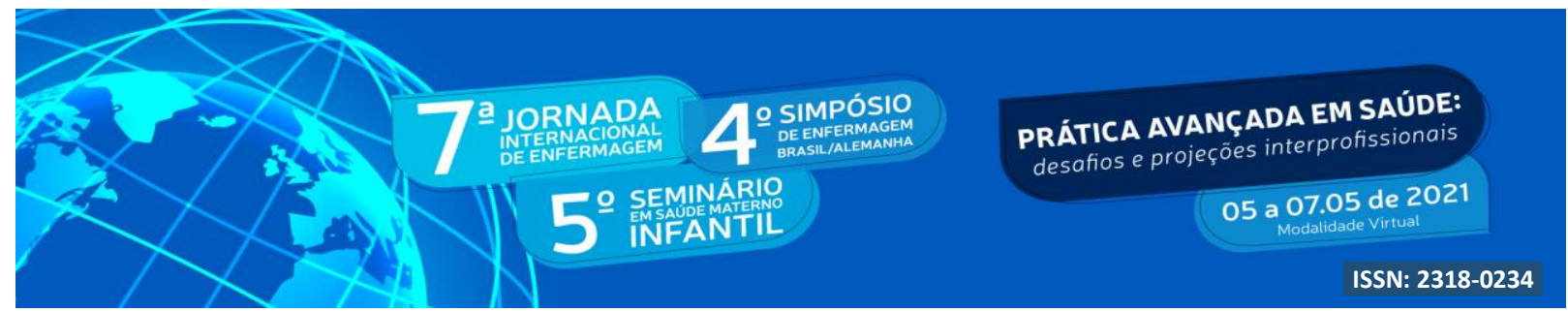

violência sexual em até 72 horas e o paciente será acompanhado pela Casa 13 de Maio para realizar exames por 90 dias. Os medicamentos estão disponíveis no Hospital Universitário de Santa Maria (HUSM), em razão de não ter uma unidade dispensadora e pela falta de profissionais farmacêuticos no município. No serviço da Casa 13 de Maio acontecem grupos de usuários com o objetivo da prevenção e promoção, capacitações com os profissionais e estudantes da rede para atingir o melhor atendimento.

Em relação ao preconceito dos profissionais foi citado pela gestora "O pessoal procura não demonstrar na hora das capacitações, mas já ouvi de profissionais que no momento de fazer o teste o profissional diz ao usuário que se não se cuidar daqui a pouquinho vai pegar aids e vai ter eu tomar coquetel, fazendo que a pessoa fique com medo". Sendo importante cuidar para não propagar discriminações e dar as informações adequadas a todos que procuram o atendimento.

Após a entrevista foi disponibilizado aos acadêmicos, folders com informações sobre as ISTs e duas caixas de preservativos e lubrificantes para serem utilizados durante o processo de intervenção.

\subsection{Detalhamento do processo de intervenção}

Com o incentivo da gestora das política e da acadêmica de enfermagem foi idealizada uma intervenção relacionada ao dia dos namorados e colocado em prática a confecção uma fantasia de preservativo, de um informativo explicativo sobre a ação em folha A4 e das caixinhas onde foram depositadas as camisinhas e os lubrificantes.

No segundo dia da vivência, dia 13 de junho de 2019, foi realizado o processo de intervenção nos banheiros públicos masculinos e femininos da Praça Saldanha Marinho, no Shopping Popular e em um bar que possuía quartos privados. Em todos os locais houve a permissão dos responsáveis para colocar em execução a atividade e os acadêmicos foram bem recebidos.

Nos banheiros públicos da Praça Saldanha Marinho, foi dialogado com o responsável pelo ambiente sobre a possibilidade de colocar os informativos e os preservativos no local. No início houve o questionamento feito por um trabalhador da limpeza, sobre a necessidade de depositar o material no local, já que segundo ele poderia incentivar a ocorrência do aumento 


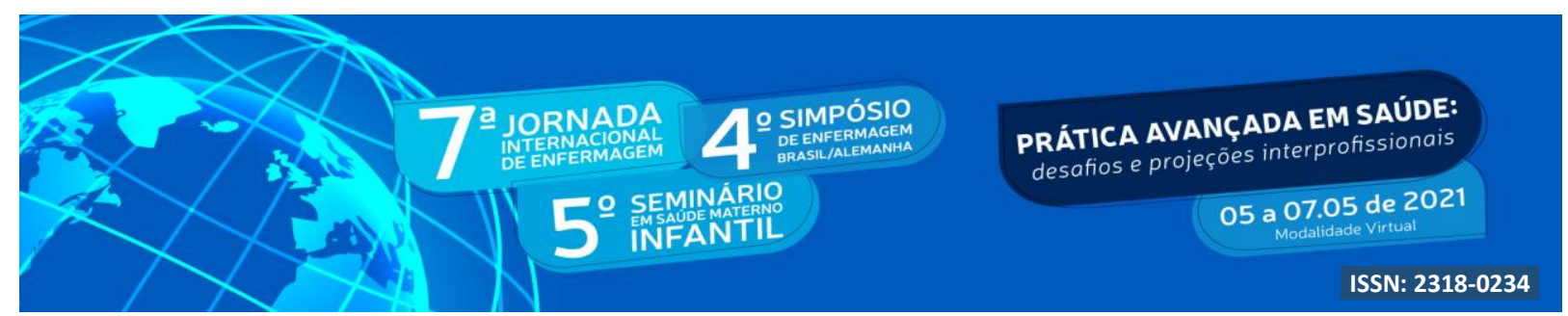

de relações sexuais dentro do banheiro.

Dessa forma, foi explicado a importância de abordar essa temática que preocupa atualmente e a necessidade da prevenção das ISTs visto que é grande a ocorrência de sexo nesse âmbito. Logo, após a autorização do responsável, os acadêmicos foram parabenizados pela iniciativa. O responsável também salientou a importância da reposição regular dos preservativos.

No Shopping Popular os acadêmicos transitaram com a fantasia pelos corredores chamando a atenção para a causa e as caixinhas foram colocadas nos banheiros. No bar com quartos privados, os acadêmicos foram recepcionados com muito entusiasmo pela responsável do estabelecimento. Ao dialogar com ela, a mesma mencionou que há falta de preservativos femininos e que foi muito significativo a realização dessa ação abrangendo as doenças e o uso de camisinhas, pois a grande procura de profissionais do sexo por homens casados e idosos nesse lugar.

\subsection{Vivências significativas por parte dos autores}

O tema exposto sobre as ISTs atualmente torna-se fundamental abordar em diversos cenários. Com a possibilidade de realizar intervenções em espaços públicos conseguimos transformar necessidades e conscientizar os indivíduos em relação à educação em saúde. Assim, ao inserir acadêmicos de graduação nessas ações é possível a ampliação do conhecimento, qualificação, formação crítica-reflexiva para lidar com a realidade e transformá-la em mudanças para o aperfeiçoamento do cuidado na vida profissional futuramente. (A1)

Ao realizar a ação no dia 13 de junho, passarmos para a população informações para a segurança e o autocuidado na utilização de preservativos nas relações sexuais. Assim, compreendemos que foi uma experiência muito gratificante e enriquecida de conhecimento visto que essas metodologias de atuação chamam a atenção das pessoas. Desse modo, essa vivência ficou marcada no processo de aprendizagem de cada aluno que participou da intervenção não só na trajetória acadêmica, mas na vida pessoal de cada um. (A2)

O conhecimento da "Políticas Nacional de DST/AIDS: Princípios e Diretrizes" e sua aplicação no município de Santa Maria-RS: as dificuldades, os planejamentos, suas 


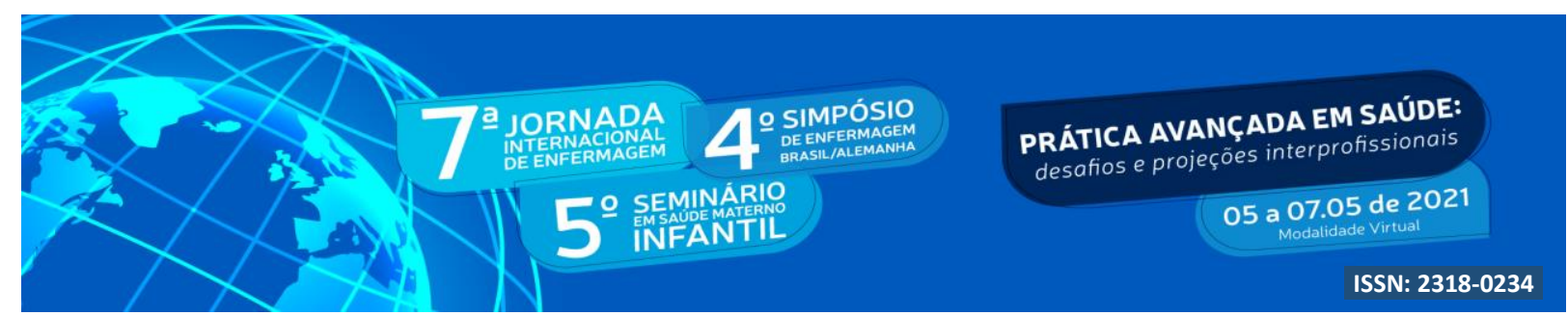

potencialidades e seu alcance ao público-alvo foram fundamentais para reconhecer a importância de ancorar a prática profissional nas estratégias do sistema único de saúde. Esse reconhecimento deve ser feito de modo integrado e as intervenções que são realizadas fora do cenário acadêmico estimulam nesse sentido. (A3)

Os acadêmicos desenvolvem as reflexões, a integração com a comunidade e a habilidade de se conceber ideias e traçar metas em equipe. $\mathrm{O}$ aprendizado adquirido possibilitou ter argumentos sobre a temática, entender a realidade social, busca por mudanças de atitudes e por conscientização, favorecendo dessa forma não só os acadêmicos envolvidos como também os sujeitos alcançados pela ação. (A3)

\section{CONCLUSÃO}

Com este trabalho concluiu-se, que a enfermagem atua em diferentes âmbitos do processo de promoção, prevenção e tratamento da saúde desempenhando um papel fundamental com a equipe multiprofissional e a população em relação às ISTs. O uso correto de preservativos, exames de rotinas, testes rápidos e uma comunicação adequada faz com que os índices de doenças diminuam.

Embora a "Política Nacional de DST/AIDS: Princípios e Diretrizes" possui recursos disponíveis e uma resolutividade em ações, percebemos fragilidades na adesão dos profissionais de saúde nas propostas de ações, o preconceito por parte da sociedade e por ser muito antiga deveria ser reavaliada e mais efetiva no modo de proporcionar o cuidado.

Nas realizações das atividades compreendemos que se torna primordial a busca de métodos que incentivem e conscientizem a população em relação à prevenção das ISTs. Dessa forma, acreditamos que a intervenção possa ter contribuído positivamente, gerando resultados gratificantes para o município de Santa Maria-RS.

\section{REFERÊNCIAS}

ARAÚJO, F.M.; SILVA, J.A.; RODRIGUES, T.S. Caracterização das infecções sexualmente transmissíveis em usuários da atenção básica: uma revisão integrativa. Revista UNINGÁ, Maringá, v.56, n.2, 2019.

\section{BRASIL. Departamento passa a utilizar nomenclatura "IST" no lugar de "DST".}




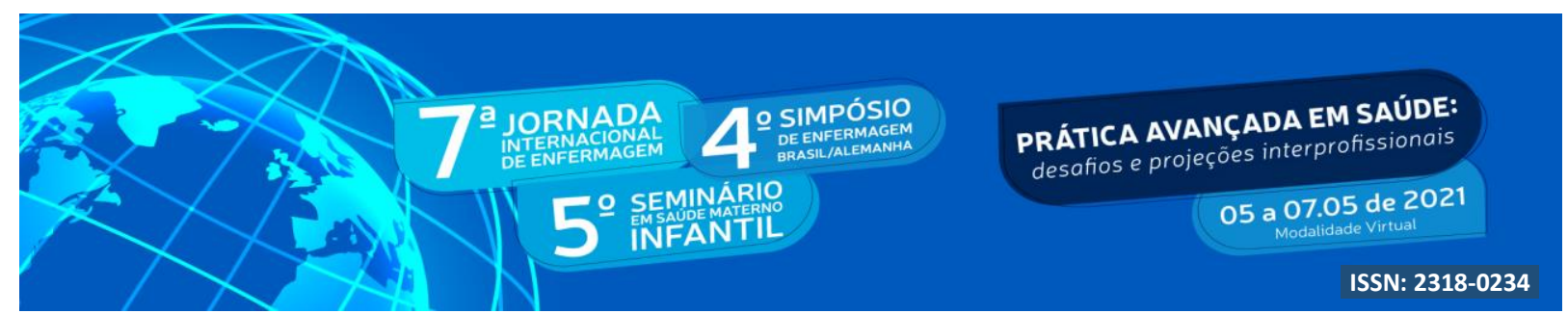

Departamento de Doenças de Condições Crônicas e Infecções Sexualmente Transmissíveis. Brasília: Ministério da Saúde, 2016.

BRASIL. Política Nacional de DST/aids: princípios e diretrizes / Coordenação Nacional de DST e Aids. Brasília: Ministério da Saúde, 1999.

FERREIRA, I.T. et al. Avaliação da qualidade da consulta de enfermagem em infecções sexualmente transmissíveis. Enfermagem Foco, v.9, n.3, 2018.

JESUS, M.I. Educação sexual com adolescentes: promovendo saúde e socializando boas práticas nas relações sociais. Pró-reitoria de pós-graduação, pesquisa e extensão na área de ciências da saúde. Mestrado Profissional em Saúde Materno Infantil. Santa Maria/RS, 2018.

KRABBE, E.C. et al. Escola, sexualidade, práticas sexuais e vulnerabilidades para as infecções sexualmente transmissíveis (IST). Revista Interdisciplinar de Ensino, Pesquisa e Extensão, v. 4 n 1 , 2016. Acesso em 06 de Junho de 2019.

SANTOS, L.F.; SILVA, J.M.; FONTELES, M.M. Educação em saúde sobre higiene íntima da mulher e infecções sexualmente transmissíveis: relato de experiência. Revista Expressão Católica Saúde; v. 2, n. 2; Jul Dez; 2017. 